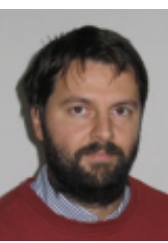

György Károlyi Associate professor, Department fructural Mechanics, Budapest Econsity

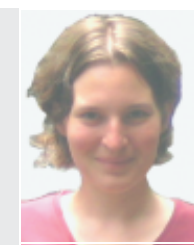

Margit Pattantyús-Ábrahám Department of Hydraulic and Water Resources Engilc and Budapest University of and Economics, Budapest, Hungary
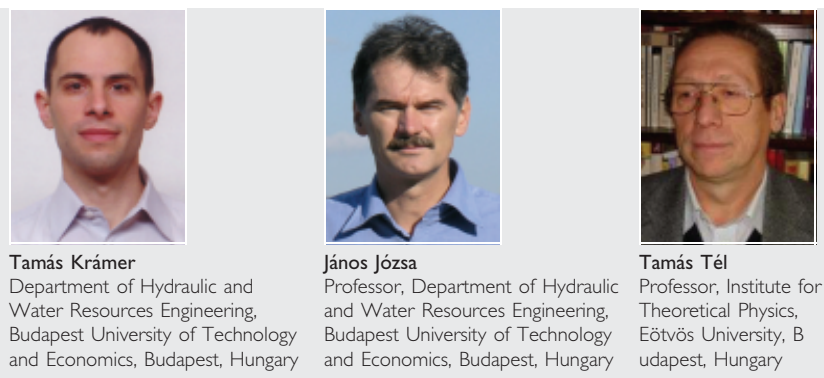

\title{
Finite-size Lyapunov exponents: a new tool for lake dynamics
}

G. Károlyi PhD, M. Pattantyús-Ábrahám PhD, T. Krámer PhD, J. Józsa PhD and T. Tél PhD

Chaotic motion of particles in fluid flows was recognised decades ago but this phenomenon has only been acknowledged recently in civil engineering. Herein it is shown that chaotic advection has a wide range of important hydraulic and environmental applications. The most important characteristics of chaotic particle transport, such as the filamentary (fractal) distribution of advected particle ensembles and their importance for active chemical or biological processes, which has farreaching ecological or environmental consequences, are reviewed. An important tool to discover and describe the filamentary distribution is the finite-size Lyapunov exponent. As a particular example, its application to an idealised shallow, wind-driven lake is presented. It is shown that the finite-size Lyapunov exponent provides a useful tool to investigate, for example, the spatial distribution of pollution in the lake - it can help to determine the most exposed shore segments and identify effectively mixed or stagnant regions in the lake.

\section{NOTATION}

$A_{0} \quad$ initial surface of area

$A_{t} \quad$ area covered by the particles at a later time $t$

$d_{0} \quad$ initial deviation

$d_{\mathrm{f}} \quad$ final separation

$\mathbf{r}(t) \quad$ position of the particle at time $t$

$t$ time

$t_{0} \quad$ the starting time

$V_{0} \quad$ initial volume covered by particles

$V_{t} \quad$ volume covered by the particles at a later time $t$,

$\mathrm{v}(\mathrm{r}, t)$ velocity field of the fluid

$\tau \quad$ time required to reach a separation $d_{\mathrm{f}}$

\section{INTRODUCTION}

Transport in fluid flows is a very important phenomenon in nature and in industry. On a large scale, the motion of pollutants (Wolff, 2008) and the formation of the ozone hole (Edouard et al., 1996; Solomon, 1999) in the atmosphere, the advection of plankton (Abraham, 1998; Abraham et al., 2000; Bracco et al., 2000; Martin, 2003; Neufeld et al., 2002) or oil spills (Károlyi and Grebogi, 2007) in oceanic flows are all examples of this phenomenon. On the smallest scale, the carrying of cells in blood flow (Schelin et al., 2009) or motion in microfluidics devices (Bottausci et al., 2004; Stroock et al., 2002) are examples of such transport. It is usually activities in the intermediate scale that are found in everyday civil engineering practice; for example, transport in rivers (Fischer et al., 1979) or mixing in lakes (Kranenburg, 1992; Liang et al., 2006, 2007; Pattantyús-Ábrahám et al., 2008).

The present study investigates the way in which the mixing of particles (such as pollutants) in lakes is characterised.

Traditionally in engineering practice mixing is associated with molecular (on a small scale) or turbulent (on larger scales) diffusion. However, in addition to diffusion, stirring by fluid motion also makes a very important contribution to mixing processes. On the smallest scales, below the Kolmogorov scale, viscosity dominates and the flow field is smooth; hence the motion of fluid parcels is governed by smooth driving imposed by the flow field. On the largest scale, diffusion is dominated by coherent flow structures such as eddies, of which the twodimensional (2-D) ones have a relatively long lifetime, and hence govern the large-scale motion. The relative importance of advection with respect to diffusion is characterised by the Péclet number $P e=L U / D$, where $L$ is the characteristic length, $U$ is the velocity scale and $D$ is the diffusion coefficient (see for example Landau and Lifshitz, 2000). In many cases relevant to civil engineering practice the Péclet number is very large and hence advection is much more important than diffusion. For example, in a lake of length $L=10 \mathrm{~km}$, with typical velocities of the order of $U=0 \cdot 1 \mathrm{~m} / \mathrm{s}$, even with an effective turbulent diffusion coefficient $D=1 \mathrm{~m}^{2} / \mathrm{s}$, it is found that $P e=1000$.

In this regime the motion of particles is governed by the fluid velocity field, namely by advection. In the simplest approximation, called passive advection (Aref, 1984; Ottino, 1989), the particles take on the velocity of the fluid instantaneously, without inertia, and the particles' motion is governed by the simple equation

$$
\mathrm{d} \mathbf{r} / \mathrm{d} t=\mathbf{v}(\mathbf{r}, t)
$$

where $\mathbf{r}(t)$ is the position of the particle at time $t$, and $\mathrm{v}(\mathrm{r}, t)$ is the velocity field of the fluid. In words, the motion of the particle is the same as the motion of a fluid element in a Lagrangian sense. If the flow is stationary, that is $\mathrm{v}(\mathrm{r}, t)=\mathrm{v}(\mathrm{r})$, the trajectories covered by the particles coincide with the streamlines of the fluid flow. However, if the velocity field depends on time, the motion of the particle deviates from the streamlines, the latter becoming an instantaneous feature of the flow field. Even a simple, smooth, periodic time dependence is 
enough for the particle trajectories to become chaotic (Aref, 1984; Ottino, 1989), and no turbulence is necessary for creating very complex particle motion.

In the next section this phenomenon, the chaotic motion of particles in fluid flows, is addressed and the most important properties of chaotic stirring are summarised. In Section 3, some applications from civil engineering are briefly discussed and Section 4 is dedicated to the results obtained from numerical simulations in a shallow lake model. In particular, the manner in which the finite-size Lyapunov exponents can be used to characterise stirring is shown. Section 5 contains further discussions and conclusions.

\section{CHAOTIC ADVECTION IN PERIODIC FLOWS}

The word chaos means, in dynamical systems theory, that the motion is irregular and sensitive to initial conditions (Tél and Gruiz, 2006). In fluid flow it implies that particles released in the flow close to each other typically deviate from each other in the course of time. This deviation is very fast: the distance between any pair of particles grows exponentially. The exponential deviation is well characterised by the Lyapunov exponents. Choosing two nearby particles at a distance $d_{0}$, their distance changes in time as

$$
2 d_{t}=d_{0} \mathrm{e}^{\lambda t}
$$

Here

$$
3 \quad \lambda\left(r, d_{0}, t_{0}, t\right)=\frac{1}{t} \ln \frac{d_{t}}{d_{0}}
$$

is the finite time or local Lyapunov exponent, which depends on the choice of the initial location $r$, the initial deviation $d_{0}$, the starting time $t_{0}$, and the time $t$ of the separation. The long time and small initial deviation limit of Equation 3 defines the Lyapunov exponents

$4 \lambda(r)=\lim _{t \rightarrow \infty} \lim _{d_{0} \rightarrow 0} \frac{1}{t} \ln \frac{d_{t}}{d_{0}}$

In particular, at each point the rate of separation can be different for different orientations of the initial separation, $d_{0}$, and it is maximum in a certain direction. When this largest Lyapunov exponent $\lambda_{1}$ is positive in almost all points at least in an extended region, the system is called chaotic.

For typical initial directions the exponential separation has the same rate, $\lambda_{1}$, after sufficient time. There are, however, exceptional directions in each point of the flow where separation has a different rate or there is convergence. The number of directions with different long time rates of separation (or convergence) is the same as the number of independent directions. In a three-dimensional fluid flow, in case of passive advection, the number of different Lyapunov exponents is thus three: $\lambda_{1}, \lambda_{2}, \lambda_{3}$.

In a similar manner to Equation 2 one can investigate how a surface changes due to the transport by the fluid. Choosing an initial surface of area $A_{0}$, covering it with particles, and measuring the area $A_{t}$, covered by the particles at a later time $t$, one finds the relation

$$
A_{t}=A_{0} \mathrm{e}^{\left(\lambda_{1}+\lambda_{2}\right) t}
$$

For long times, this relation defines the second largest Lyapunov exponent $\lambda_{2}$. Similarly, covering an initial volume, $V_{0}$, by particles and measuring the volume $V_{t}$, covered by the particles at a later time $t$, the smallest Lyapunov exponent, $\lambda_{3}$, can be defined by the relation

\begin{tabular}{|l|l|}
\hline & $V_{t}=V_{0} \mathrm{e}^{\left(\lambda_{1}+\lambda_{2}+\lambda_{3}\right) t}$ \\
\hline
\end{tabular}

In incompressible fluids, the total volume does not change and hence the sum of the three Lyapunov exponents must be zero: $\lambda_{1}+\lambda_{2}+\lambda_{3}=0$.

In case of passive advection in 2-D fluid flows, as in shallow lakes or in layered fluids, there are only two Lyapunov exponents. Typically, the largest Lyapunov exponent, $\lambda_{1}$, has the most important role. Indeed, this governs the rate of exponential divergence; this term will determine the growth described by Equation 2. In incompressible planar flow the smaller Lyapunov exponent, $\lambda_{2}$ is just the negative of the larger one, $\lambda_{2}=-\lambda_{1}$

If the Lyapunov exponents are not zero in a region of the 2-D flow, that is, $\lambda_{2}<0<\lambda_{1}$, there is a strong stretching in the fluid. Injecting many particles into the fluid in a compact patch, like a blob of dye, the patch is strongly stretched along the fastest separating direction at each point. The particles eventually accumulate along curved lines, called filaments. The fastest separating direction is typically different at each point, which results in a highly distorted filament as the shape of the blob of particles. Along these lines the particles diverge from each other exponentially due to the positive Lyapunov exponent, $\lambda_{1}>0$, but particles from further away approach these filaments due to the negative Lyapunov exponent, $\lambda_{2}<0$. In other words, the particles accumulate along these complicatedly winding curves after a short time. Chaos is always associated with a strong stretching and folding action, and this manifests itself in a quick alignment of passively advected particles along filaments. In dynamical systems jargon these filaments are called the unstable manifold of the chaotic set, the latter containing the unstable periodic particle orbits (Tél and Gruiz, 2006). The name unstable manifold indicates that behaviour along them is very unstable due to the exponential separation. Such filaments are easily observable in streams or lakes (see Figure 1 for an example), and one can also see them while stirring cream in coffee. Due to the exponential separation of initially nearby particles, mixing is very fast in these chaotic regions.

In regions of the fluid where the local Lyapunov exponents are zero (or, in practice, close to zero), the exponential separation does not occur. These regions are often associated with eddies, gyres or vortices in the fluid, because in many cases these nonchaotic regions can be associated with vortical flow structures. However, these non-chaotic regions are not immediately 


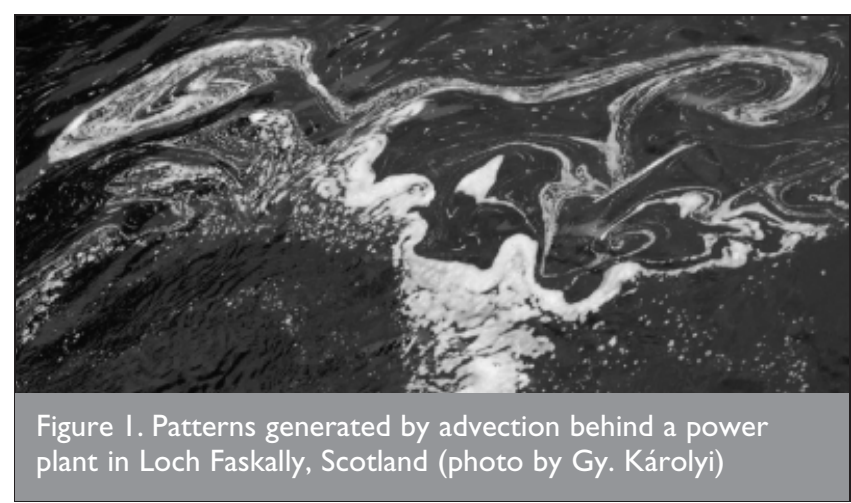

connectable to Eulerian characteristics of the fluid - the particle orbits in a non-stationary flow are not the same as the streamlines. Anyway, mixing in the non-chaotic regions is much slower than in the chaotic regions.

Chaotic and non-chaotic regions are separated from each other by impenetrable boundaries, although the boundaries can wander in time. In practice, it is very important to distinguish between chaotic and non-chaotic regions of the flow. For example, if one is interested in how pollution is distributed by fluid stirring, it is of interest to know in which region the source is located - whether it is in the chaotic or in the nonchaotic region of the flow. Identifying the vortex boundaries and barriers to transport has already been identified as a major step in understanding mixing in fluid flows (Boffetta et al., 2001; d'Ovidio et al., 2004; Haller, 2000; Iudicone et al., 2002).

\section{APPLICATIONS IN CIVIL ENGINEERING}

Horizontal stirring is of particular importance in shallow environmental flows. Its understanding and accurate modelling are essential for water exchange mechanisms, interpreting plankton movements, or planning and operating pollutant outfalls. In order to understand the properties of the particle transport, the Eulerian description of the fluid velocity field is not enough. One has to apply a Lagrangian description, which gives more direct information on particle transport. The connection between the Eulerian and Lagrangian descriptions of fluid motion is not trivial, and if one wants to get information on the particle distribution, the Lagrangian approach is the natural one.

As a particular civil engineering example, Liang et al. (2009) have investigated shallow water covers of reactive sulfide mine tailings produced by mining and milling of metallic ores (e.g. uranium). The wind-driven resuspension of the environmentally hazardous materials may occur in stormy weather, and they investigated the transport and chemical activity of the hazardous material in the lake. They found that the effects of chaotic advection on the chemical activity follow closely the theoretical findings by Károlyi and Tél (2005).

Looking at wind-driven sediment transport in shallow lakes, a more complete description comprises resuspension, horizontal transport and deposition, as a combined effect of flow and waves. In such a system horizontal advection of suspended solids would be accompanied by a source and sink term, the two latter modifying, but not cancelling, the effects of chaos.

Another very important and up-to-date field is the investigation of the effects of climate change on transport processes in fluids, be it oceans (Toggweiler and Russell, 2008), lakes (Lam and Schertzer, 1999) or rivers (Whitehead et al., 2009). A very important effect of climate change is on the water level dynamics of lakes. These effects are most pronounced in shallow lakes, where small fluctuations in water level result in significant changes in the depth, thus having important consequences for transport processes. In the next section the effect of water level change on the horizontal stirring in a shallow lake is investigated.

It has been shown (Károlyi et al., 2000; Scheuring et al., 2000) that the filamentary patterns generated by chaotic fluid motion have a very important effect on aquatic populations. For instance, phytoplankton populations trace out filamentary structures, most remarkably during plankton blooming (Abraham 1998; Abraham et al., 2000; Bracco et al., 2000; Martin, 2003; Neufeld et al., 2002). Zooplankton, that are able to actively swim while grazing on phytoplankton, follow a similar distribution (Sandulescu et al., 2008), just like top marine predators (Tew Kai et al., 2009). The filamentary distribution has a very important role in the biological activity of plankton. In fact, it has been suggested as a possible mechanism that explains the paradox of plankton. According to traditional population biological equations assuming that plankton is homogeneously mixed in the water, the number of coexisting species cannot exceed the number of limiting resources. This means that the number of coexisting species in a well-mixed environment cannot exceed, say, 10. That this is evidently not the case for plankton population can be explained by the non-homogeneous mixing caused by the chaotic motion in fluid flows that generate filamentary distribution of plankton (Károlyi et al., 2000; Scheuring et al., 2000).

Horizontal fluid stirring, as will be seen in the next section, is characterised by a filamentary distribution of particles. It is very important to know where the filamentary structures, the unstable manifolds reside, as they govern the properties of stirring in fluids. In order to investigate the emergence of the filamentary structures, a simple but realistic wind-driven shallow water model is investigated in the following section.

\section{FINITE-SIZE LYAPUNOV EXPONENTS IN A SHALLOW LAKE}

In order to investigate the particle motion in a realistic fluid flow, the velocity field in a wind-driven shallow lake is computed numerically. For simplicity, the lake has a square shape of horizontal dimensions $2 \mathrm{~km} \times 2 \mathrm{~km}$, and its depth is varied linearly between $2 \mathrm{~m}$ at the shore and $2.5 \mathrm{~m}$ in the centre (Figure 2). The lake is forced by a wind of velocity components $10 \mathrm{~m} / \mathrm{s}$ in both north and either east or west directions, with the wind changing direction at every $4 \mathrm{~h}$ from north-east to north-west and vice versa. The transition in the wind direction takes 20 min - during this time the north component is kept constant while the east component decays to zero and the west component increases or vice versa. This kind of forcing is reminiscent of the one used by Kranenburg (1992) and Liang et al. (2006, 2007), and results in a highly unsteady periodic flow of period $T=8 \mathrm{~h}$. The near-surface wind and the surface shear stress field are estimated considering an atmospheric internal boundary layer over the 


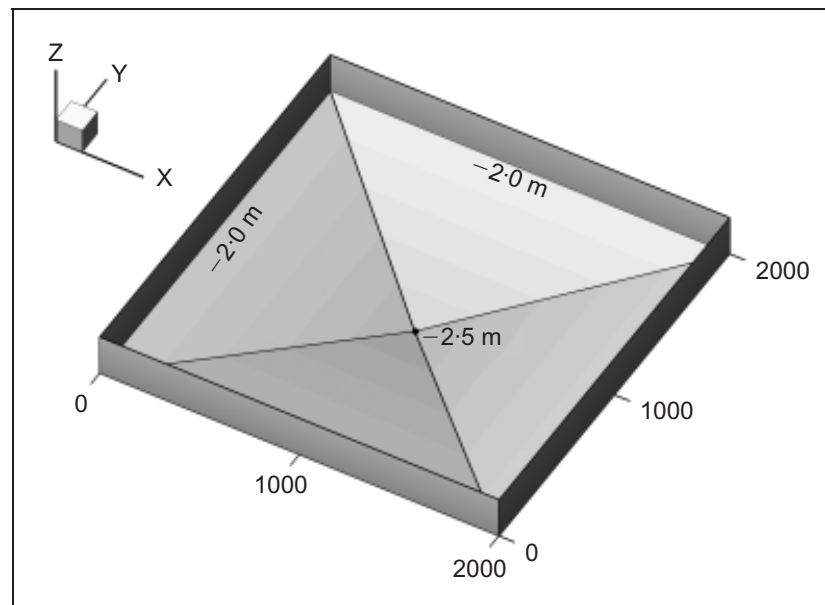

Figure 2. Axonometric view of the model lake geometry

water surface using semi-empirical formulae justified by field data and numerical modelling (Curto et al., 2006; Józsa et al., 2007). In sufficiently strong wind conditions the horizontal water mass exchange in a shallow lake can be reasonably approximated in a depth-integrated horizontal manner, allowing the use of the 2-D shallow water equations for the numerical flow simulation (Curto et al., 2006; Vreugdenhil, 1994). A uniform Manning bed roughness of $n=0.025 \mathrm{~s} / \mathrm{m}^{1 / 3}$ is assumed and the effects of Earth's rotation are neglected, due to the moderate extent of the lake. As boundary conditions, no normal flow is allowed along the shore, and there is perfect slip of the tangential flow component. The numerical solution is obtained using a standard, spatially second-order accurate finite difference method (Vreugdenhil, 1994) on a rectangular grid of $40 \mathrm{~m}$ cell size. The flow state variables are assigned to this grid according to the Arakawa C layout: the components of the specific discharge are represented at cell edges whereas the water depth is cell-centred. The time integration is done using an explicit Euler scheme with a time step of the order of 10 s governed by the Courant stability condition. The Eulerian velocity components obtained on this grid are stored at time steps of $600 \mathrm{~s}$ for the particle trajectory calculations; see for example the left image in Figure 3. Then the advection Equation 1 is integrated by a fourth-order Runge-Kutta scheme using the velocity components obtained by bilinear spatial interpolation within the grid cells and by linear interpolation between the stored time levels.

The definition (Equation 4) of the Lyapunov exponents involves that the rate of separation needs to be measured for very long times. This is not feasible either in numerical or real experiments. Also, in practice the limit $d_{0} \rightarrow 0$ cannot be effectively measured. Instead, the concept of finite-size Lyapunov exponents (FSLE) has been introduced (Aurell et al., 1997; Boffetta et al., 2001). To measure the FSLE at a point $r$, a reference particle is started from $r$ at time $t$, simultaneously with another particle at a distance $d_{0}$ from $r$. The time $\tau$ required to reach a separation $d_{\mathrm{f}}$ is measured, and the FSLE is defined as

$$
\lambda\left(r, t, d_{0}, d_{\mathrm{f}}\right)=\frac{1}{\tau} \ln \frac{d_{\mathrm{f}}}{d_{0}}
$$

Large values of the FSLE mark regions in the flow where neighbouring particles are subjected to large stretching, where dependence on the initial conditions is sensitive, hence where chaos reigns. Figure 4 shows a plot, for different initial times $t$, of the FSLE distribution in the model lake obtained by releasing $381 \times 381$ particles in total, in the nodes of a regular grid of $5 \mathrm{~m}$ resolution, excluding the $50 \mathrm{~m}$ wide littoral belt. For the calculations, in each of the grid points the average separation of the four initially diagonally adjacent particles are used $\left(d_{0}=\sqrt{2} \cdot 5 \mathrm{~m}\right)$ and the average time $\tau$ for the separation to reach $d_{\mathrm{f}}=\sqrt{2} \cdot 250 \mathrm{~m}$ is measured.

\section{As Figure 4 shows, the high values in the FSLE distribution} trace out filamentary structures. The filaments are the results of the strong stretching and folding characteristic to chaotic systems (Tél and Gruiz, 2006). They indicate the unstable manifold - that is, the largest stretching direction in the fluid. Between the filaments of high FSLE values there are 'valleys' of lower values. This means that nearby points can have quite different FSLE values if they are lying on different filaments. There are extended regions, however, where the FSLE values are low (close to zero): these indicate regions with poor mixing. As can be seen from Figure 4, the shape of the filaments and the shape of the region with low FSLE change with time. For example, the low FSLE regions seem to be rotating. Despite this time-dependence, between regions of strong and weak mixing there is no exchange by stirring, there is a barrier to transport. Therefore, it is of great importance to distinguish between the chaotic and non-chaotic regions. For example, if pollution is injected in a region of poor mixing, it remains there for a long time. However, pollution injected in a chaotic region spreads rapidly in the whole chaotic domain due to the high stretching rates (large FSLE).

The extent of the regions with filamentary structures indicates the strength of chaos. The size of the chaotic, well-mixed regions of the flow depends in a non-trivial way on the Eulerian characteristics of the flow. Changes in the environment (such as water depth through varying precipitation or evaporation), alterations in land usage around the lake (through roughness that changes the wind forcing field over the lake) are factors that modify the flow field in the lake, namely the Eulerian characteristics of fluid motion. The modifications to the flow field in turn alter the Lagrangian patterns traced out by the advected particles.

\section{I. Effect of water level decrease}

To verify the effects of the environment on the filamentary structures further simulations were carried out. Figure 5 shows the FSLE distribution when the water level is decreased by $1 \mathrm{~m}$. In the shallow model lake, a $1 \mathrm{~m}$ decrease of water level enhances the relative depth between various parts of the lake: in this case the depth varies between 1 and $1.5 \mathrm{~m}$. This implies a difference in the flow field (Figure 3), which manifests itself in a different FSLE distribution (Figure 5). It can be seen from Figure 5 that filamentary structures emerge in this case as well as in the original case. However, the patterns traced out by the filaments are different. An interesting difference is that in the shallower case regions of low mixing cannot be seen: the filaments visibly cover the whole lake. This is true even though vortical structures are still present in the Eulerian velocity field (Figure $3 b$ ). In fact, unchanged wind forcing imposed on a lake 


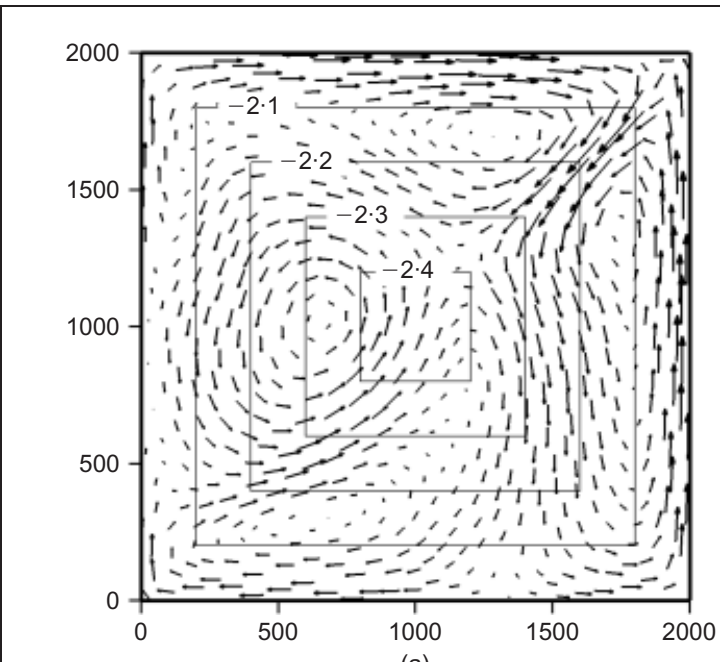

(a)

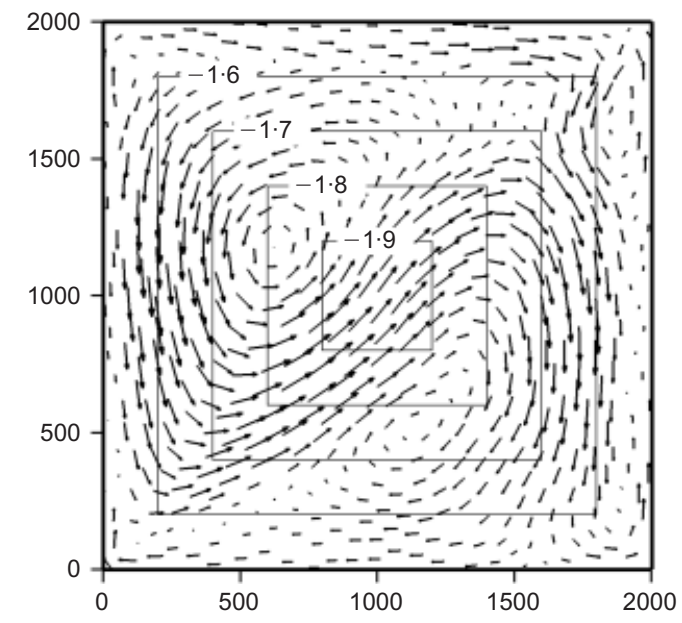

$0.1 \mathrm{~m} / \mathrm{s}$

Figure 3. Instantaneous flow pattern in the model lake with (a) original and (b) decreased water level at time $t=T$
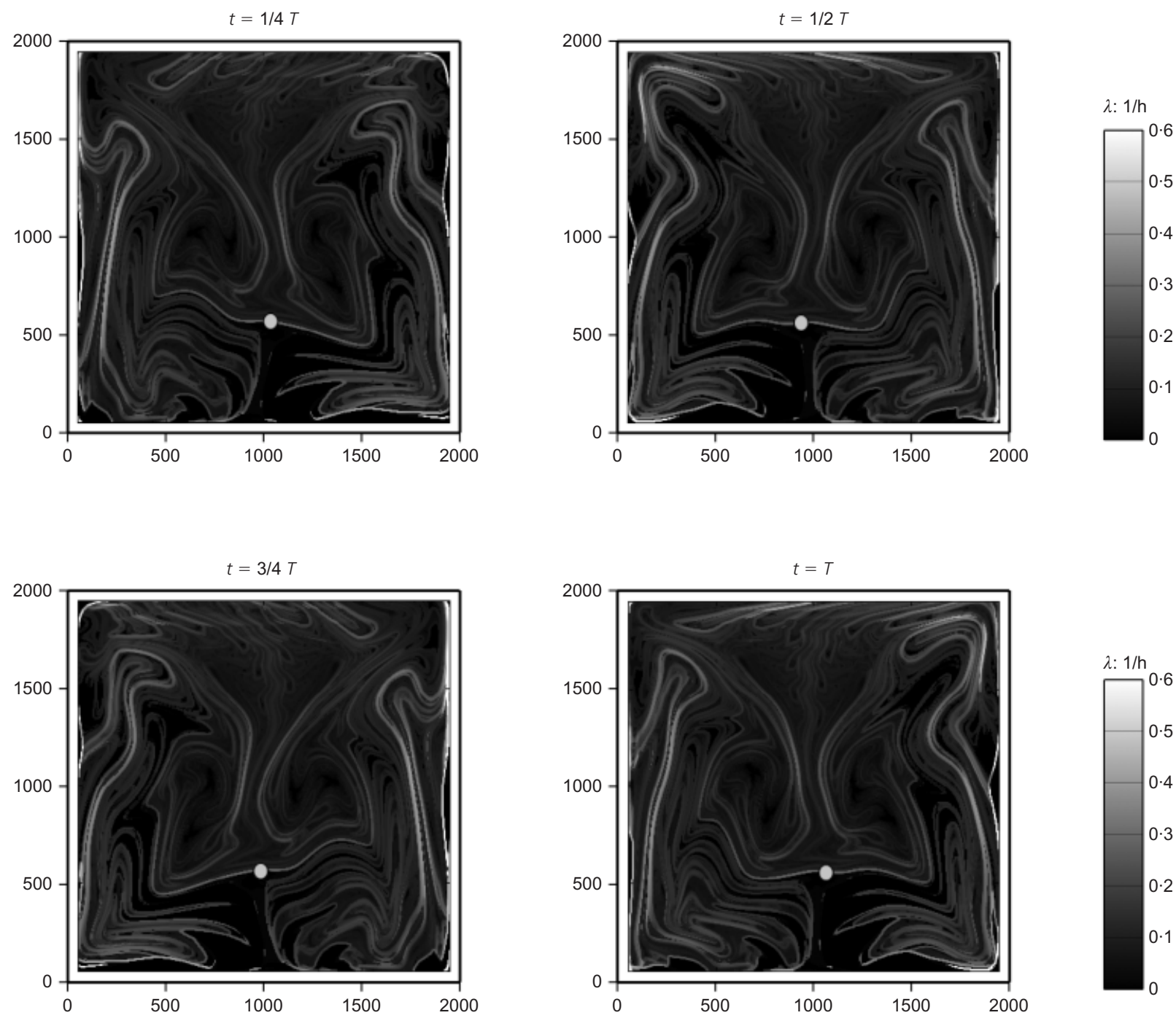

Figure 4. The FSLE distribution in the model lake with different initial times $t$ as a fraction of the period $T=8 \mathrm{~h}$. The shading indicates the FSLE values in units of $\mathrm{I} / \mathrm{h}$, obtained with initial and final separation distances of $d_{0}=\sqrt{2} \cdot 5 \mathrm{~m}$ and $d_{\mathrm{f}}=\sqrt{2} \cdot 250 \mathrm{~m}$, respectively. Starting with the lake at rest, 45 full wind periods were simulated before the FSLE calculation. Grey circle indicates instantaneous position of a periodic particle orbit 

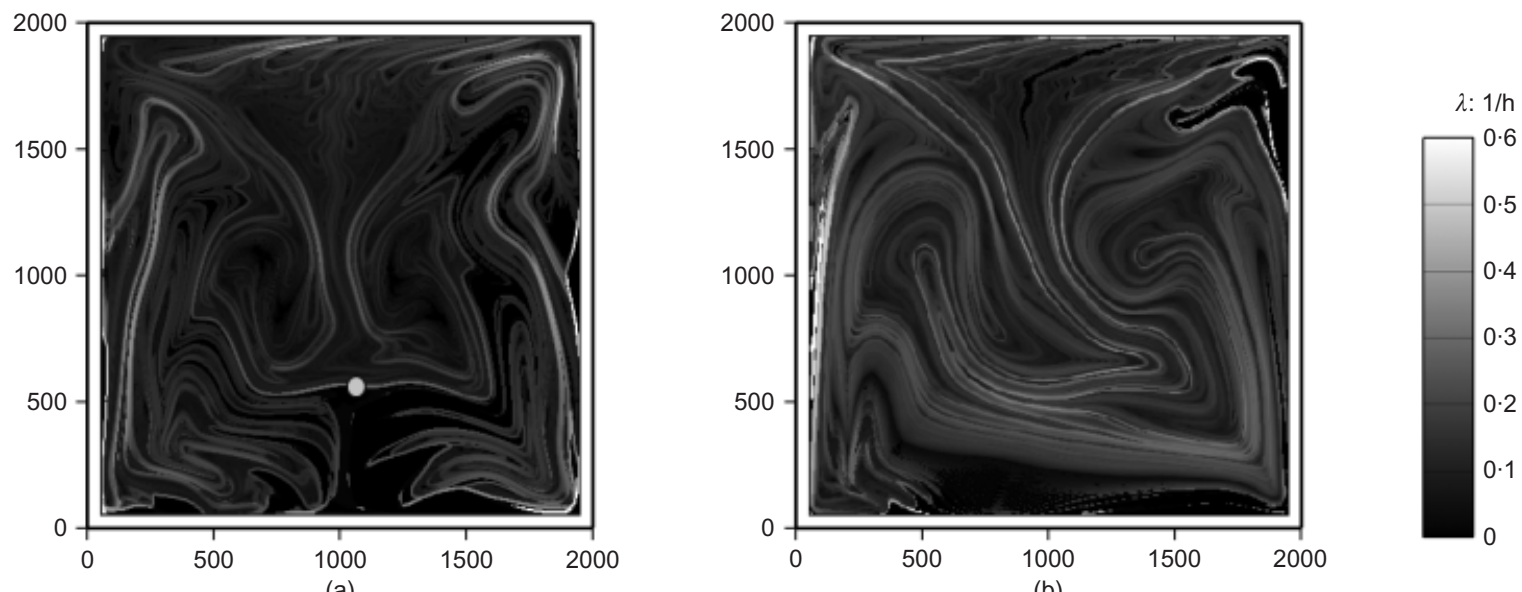

Figure 5. The FSLE distribution in the model lake with (a) original water level and (b) water level decreased by I $\mathrm{m}$. The shading indicates the FSLE values in units of $I / \mathrm{h}$, obtained with initial and final separation distances of $d_{0}=\sqrt{2} \cdot 250 \mathrm{~m}$ and

$d_{f}=\sqrt{2} \cdot 250 \mathrm{~m}$, respectively. The snapshots were taken at $t=T$

with overall reduced depth and at the same time enhanced relative depth gradients results in stronger rotation of the lakewide large gyres. It implies a larger displacement of particles in one period, the alternation of which will then generate enhanced, more extended regions with chaotic stirring. The mean FSLE averaged over the whole lake was compared and found to be $0 \cdot 11851 / \mathrm{h}$ for the original case, and $0 \cdot 16221 / \mathrm{h}$ for the case with decreased water level. This confirms that chaos and hence mixing is stronger when water level is decreased.

\subsection{Effect of coastal land use}

Figure 6 shows how land use can modify the particle transport in the lake. The relevant changes are those that occur upwind, since they also alter the velocity profile and, in consequence, the shear stress distribution over the lake. In the original case, the shore was assumed to be smooth, for example bare land with aerodynamic roughness height $z_{0}=0.005 \mathrm{~m}$. If the shore or the littoral zone is assumed to be covered by reed (Figure $6(\mathrm{a}), z_{0}=0 \cdot 15 \mathrm{~m}$ ) or by tall trees or buildings (Figure 6(b), $z_{0}=0.5 \mathrm{~m}$ ), the overland aerodynamic roughness increases, which ultimately alters the flow velocity field in the lake (Józsa et al., 2007). Again it is observed that the filamentary structures are different in the case of the reed-covered (Figure 6(a) panel) or built-in (Figure 6(b) panel) shore from that in the case of less rough shore, but the large FSLE values still trace out filamentary patterns. As the roughness of the shore increases, the wind-shear unevenness also increases, which results in higher values of the FSLEs: the measured average FSLE values are $0 \cdot 11851 / \mathrm{h}$ (original), $0 \cdot 15791 / \mathrm{h}$ (reed covered) and $0 \cdot 19501 / \mathrm{h}$ (built-in or woody shore). Furthermore, as the shore roughness increases, the non-mixing regions are gradually destroyed as chaotic stirring extends to the whole lake.

\subsection{Non-periodic wind forcing}

So far it has been assumed that wind direction is perfectly periodic, the period being $8 \mathrm{~h}$. This is, obviously, never the case for real winds. Now a check is made of what happens if the wind direction changes between north-east and north-west with partly random periodicity. The length of the period is varied between 4 and $12 \mathrm{~h}$ randomly with uniform probability (see Figure 7 for a sample time series). Despite the non-periodic

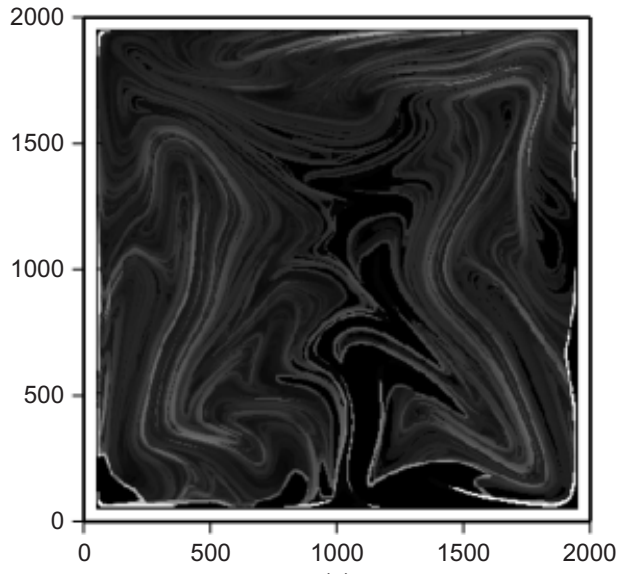

(a)

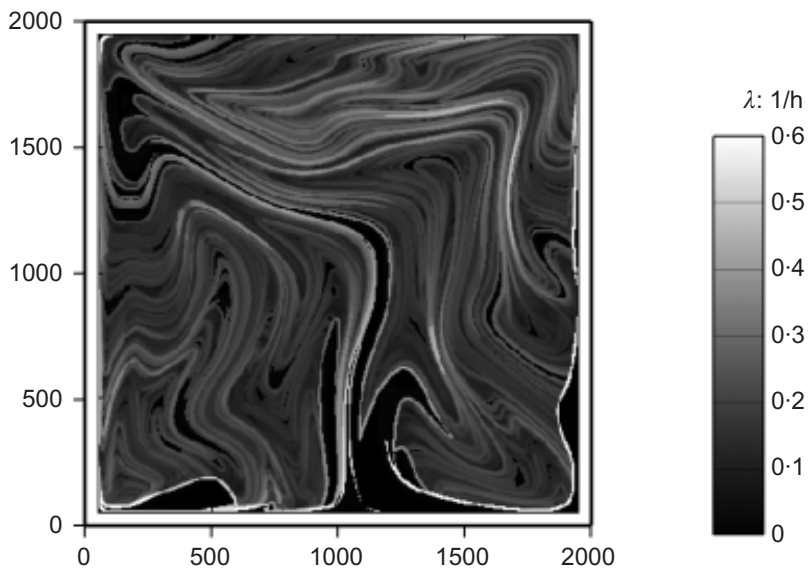

(b)

Figure 6. FSLE distribution in the model lake with rough reed-covered shore (a) (aerodynamic roughness height of $\mathrm{z}_{0}=0.15 \mathrm{~m}$ ) and even rougher built-in or woody shore $(b)\left(z_{0}=0.5 \mathrm{~m}\right)$. The shading indicates the FSLE values in units of $I / \mathrm{h}$, obtained with initial and final separation distances of $d_{0}=\sqrt{2} \cdot 5 \mathrm{~m}$ and $d_{\mathrm{f}}=\sqrt{2} \cdot 250 \mathrm{~m}$, respectively. The snapshots were taken at $t=T$ 


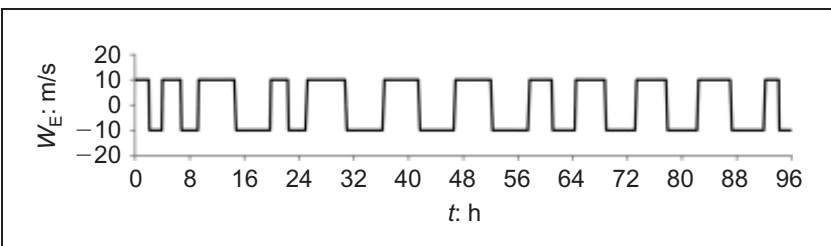

Figure 7. Non-periodic variation of the easterly component of the wind vector in time, with the period $T$ randomly

perturbed in the range 4 to $12 \mathrm{~h}$. The northerly component is held constant at $W_{\mathrm{N}}=10 \mathrm{~m} / \mathrm{s}$

wind forcing, very little change in the FSLE distribution can be seen in Figure 8 in comparison with Figure 4, in particular, the filamentarity remains intact. There is no visible change in the width of the filaments, and the high FSLE values are to be found roughly at the same locations in both figures. This shows that the filamentary distribution is not an artifact of the periodicity of the model. There is one important change, however: the FSLE patterns do not repeat in time with any period. This nonperiodicity is responsible for the lack of non-mixing regions: despite having extended regions of low mixing, the particles can escape the low FSLE regions after a long time.

\subsection{Pollutant behaviour}

A very important application of the FSLE is that it allows for the prediction of the behaviour of pollutants. Pollutants that are initiated inside a low FSLE region are expected to persist there for a long time without much dispersion. However, if the pollution starts in the chaotic regime, where the FSLE are large, it will quickly spread in the whole chaotic domain. As an example, Figure 9 shows how pollution reaches near-shore regions originating from different parts of the lake. The four shades of grey indicate which near-shore region is visited first from a point inside the lake. White regions indicate particles that never approach any of the near-shore regions. By comparing Figures 9 and 4 it can be seen that the regions from where the pollution never reaches the shore coincide with the low FSLE regions, whereas particles with high FSLE values are drifted to one of the shores. Which shore is visited by a particle is highly unpredictable due to the chaotic motion and the sensitivity to the initial location in these regions. Anyway, the

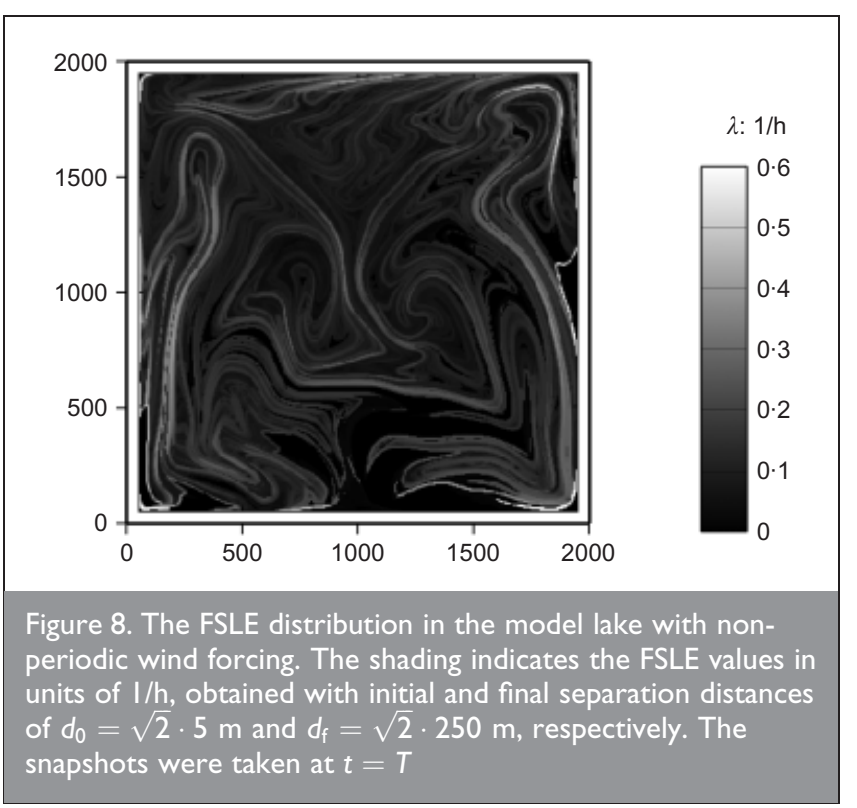

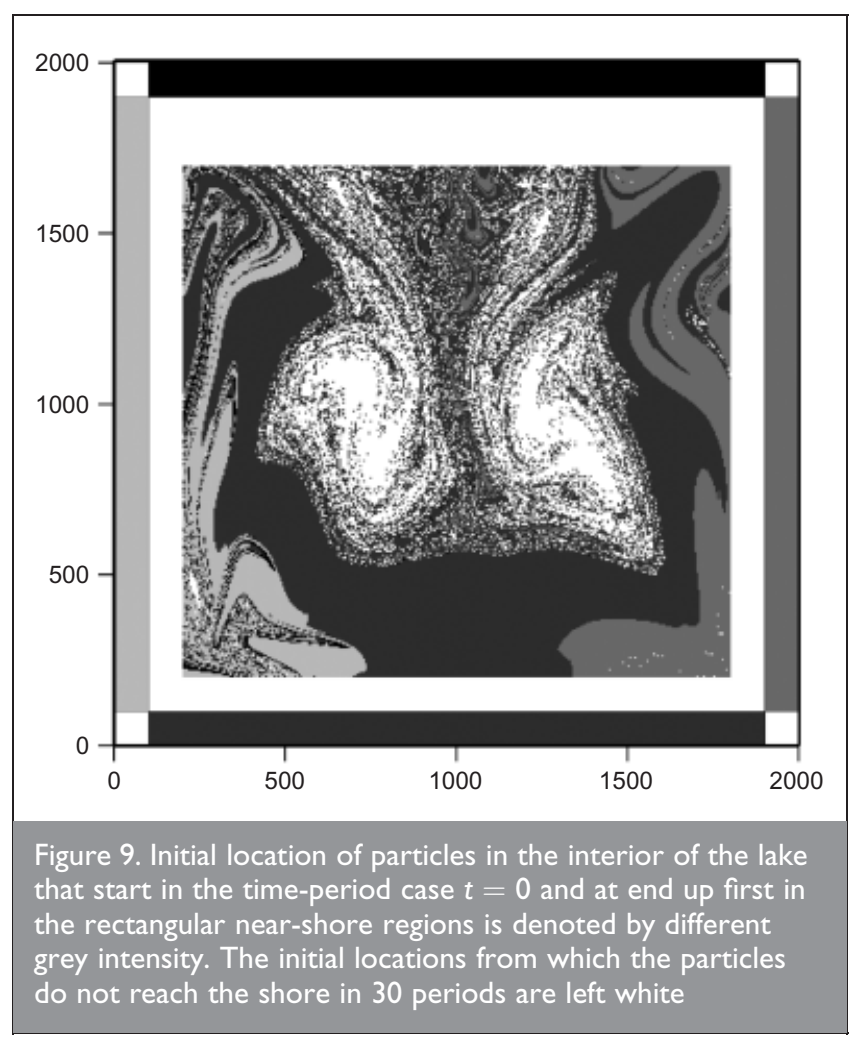

unpredictable regions coincide with the regions of high FSLE values.

\section{FURTHER OUTLOOK AND CONCLUSIONS}

In the present study some further understanding of the importance of the filamentary distribution of passive particles transported in fluid flows has been obtained. As an illustrative example, a simple wind-driven shallow lake model was investigated to see whether strong mixing is associated with chaotic motion of particles. The regions with large deviation between particles trace out filamentary patterns, that can be visualised using the finite-size Lyapunov exponents. As a very important application, it has been shown that the unpredictability of where a patch of pollutant reaches the shore depends largely on the stirring properties in the lake, which can be well characterised by the FSLE distribution. The FSLE has predictive power on whether the pollution remains inside a non-chaotic region or reaches the shore.

The manner in which large-scale environmental changes affect the mixing properties in the lake was also investigated. Whether it is the change of water level or the change in land use around the lake, the filamentary distribution of the FSLE remains. However, the exact distribution of the strongly mixed regions can change dramatically due to external effects, which is very instructive in the light of current climate change. For example, warming can decrease the level of the lake and change the vegetation or land use around the lake, leading to a dramatic effect on stirring and hence on aquatic ecosystems.

The effect of diffusion on the FSLE distribution has been studied by Pattantyús-Ábrahám et al. (2008). It was found that the combined effect of diffusion and stretching does not alter the existence of the filamentary structures. Furthermore, in the presence of diffusion (either molecular in small scales or turbulent in large scales) the barriers between strongly and 
poorly stirred regions become penetrable, but in the case of large Péclet number the water exchange remains slow. It was also found that the structure of the FSLE distribution is unchanged as the resolution of the applied numerical grid is increased. With an enhanced resolution, however, the finer details of the filaments emerge.

Throughout this study several simplifying assumptions were made on the investigated aero- and hydrodynamic conditions (wind having only two steady directions, two-dimensionality, etc.) and on the transport of particles (being passive). Previous theoretical studies (Tél et al., 2005) indicate, however, that the results outlined herein are not expected to be altered significantly by including more precise models for either the lake or the particles' transport, such as considering the effects of three-dimensionality, more random wind forcing or the inertia of particles.

Finally, it is noteworthy that an alternative approach to visualise the filamentary structures has been suggested by Haller (2000), Voth et al. (2002) and Mathur et al. (2007), which finds finite time stable and unstable manifolds. In fact, the FSLE implemented in the current case is easier to compute numerically, although the two techniques lead to essentially the same result.

\section{ACKNOWLEDGEMENTS}

This paper is dedicated to the memory of Professor Gerhard H. Jirka, a pioneer of the science of environmental fluid mechanics. The authors thank OTKA for its financial support under grant No. NK 72037.

\section{REFERENCES}

Abraham ER (1998) The generation of plankton patchiness by turbulent stirring. Nature 391(6667): 577-580.

Abraham ER, Law CS, Boyd PW, Lavender SJ, Maldonado MT and Bowie AR (2000) Importance of stirring in the development of an iron-fertilised phytoplankton bloom. Nature 407(6805): 727-730.

Aref H (1984) Stirring by chaotic advection. Journal of Fluid Mechanics 143: 1-21.

Aurell E, Boffetta G, Crisanti A, Paladin G and Vulpiani A (1997) Predictability in the large: an extension of the concept of Lyapunov exponent. Journal of Physics A 30(1): $1-26$.

Boffetta G, Lacorata G, Redaelli G and Vulpiani A (2001) Detecting barriers to transport: a review of different techniques. Physica D 159(1-2): 58-70.

Bottausci F, Mezic I, Meinhart CD and Cardonne C (2004) Mixing in the shear superposition micromixer: threedimensional analysis. Philosophical Transactions of the Royal Society of London A 362(1818): 1001 - 1018.

Bracco A, Provenzale A and Scheuring I (2000) Mesoscale vortices and the paradox of the plankton. Proceedings of the Royal Society of London B 267(1454): 1795-1800.

Curto G, Józsa J, Napoli E, Krámer T and Lipari G (2006) Large scale circulations in shallow lakes. In Advances in Fluid Mechanics. Vorticity and Turbulent Effects in Fluid Structure Interactions, vol. 45 (Brocchini $\mathrm{M}$ and Trivellato $\mathrm{F}$ (eds)). Wessex Institute of Technology Press, Southampton, UK, pp. 83-104.
d’Ovidio F, Fernández V, Hernández-García E and López C (2004) Mixing structures in the Mediterranean Sea from finite-size Lyapunov exponents. Geophysical Research Letters 31: L17203.

Edouard S, Legras B, Lefèvre F and Eymard R (1996) The effect of small-scale inhomogeneities on ozone depletion in the Arctic. Nature 384(6608): 444-447.

Fischer HB, List EJ, Koh RCY, Imberger J and Brooks NH (1979) Mixing in Inland and Coastal Waters. Academic Press, New York, USA.

Haller G (2000) Finding finite-time invariant manifolds in twodimensional velocity fields. Chaos 10(1): 99-108.

Iudicone D, Lacorata G, Rupolo V, Santoleri R and Vulpiani A (2002) Sensitivity of numerical tracer trajectories to uncertainties in OGCM velocity fields. Ocean Modelling 4(3-4): 313-325.

Józsa J, Milici B and Napoli E (2007) Numerical simulation of internal boundary-layer development and comparison with atmospheric data. Boundary-Layer Meteorology 123(1): 159175.

Károlyi G and Grebogi C (2007) Chaotic advection and fractality: applications in oceanography. Proceedings of the Oceans'07 IEEE Conference, Aberdeen, UK, 1-5.

Károlyi G and Tél T (2005) Chemical transients in closed chaotic flows: the role of effective dimensions. Physical Review Letters 95(26): 264501.

Károlyi G, Péntek Á, Scheuring I, Tél T and Toroczkai Z (2000) Chaotic flow: the physics of species coexistence. Proceedings of the National Academy of Sciences 97(25): 13661-13665.

Kranenburg C (1992) Wind-driven chaotic advection in a shallow model lake. Journal of Hydraulic Research 30(1): 29-46.

Lam DCL and Schertzer WM (eds) (1999) Potential Climate Change Effects on Great Lakes Hydrodynamics and Water Quality. ASCE, Reston, VA, USA. p. 232.

Landau LD and Lifshitz EM (2000) Fluid Mechanics. Elsevier, Butterworth-Heinemann, Oxford, UK.

Liang Q, Borthwick AGL and Taylor PH (2006) Wind-induced chaotic advection in shallow flow geometries. Part I, Circular basins; Part II, Non-circular basins. Journal of Hydraulic Research 44(2): 170-179 and 180-188.

Liang Q, Borthwick AGL and Taylor PH (2007) Particle mixing and reactive front motion in unsteady open shallow flow modelled using singular value decomposition. Computers and Fluids 36(2): 248-258.

Liang Q, Taylor PH and Borthwick AGL (2009) Particle mixing and reactive front motions in chaotic but closed shallow flows. Computers and Fluids 38(2): 382-392.

Martin AP (2003) Phytoplankton patchiness: the role of lateral stirring and mixing. Progress in Oceanography 57(2): 125174.

Mathur M, Haller G, Peacock T, Ruppert-Felsot JE and Swinney HL (2007) Uncovering the Lagrangian skeleton of turbulence. Physical Review Letters 98(14): 144502.

Neufeld Z, Haynes PH, Garcon V and Sudre J (2002) Ocean fertilization experiments may initiate a large scale phytoplankton bloom. Geophysical Research Letters 29: 1534.

Ottino JM (1989) The Kinematics of Mixing: Stretching, Chaos and Transport. Cambridge University Press, Cambridge.

Pattantyús-Ábrahám M, Tél T, Krámer T and Józsa J (2008) Mixing properties of a shallow basin due to wind-induced 
chaotic flow. Advances in Water Resources 31(3): 525-534. Sandulescu M, López C, Hernández-García E and Feudel U (2008) Biological activity in the wake of an island close to a coastal upwelling. Ecological Complexity 5(3): 228-237.

Schelin AB, Károlyi G, de Moura APS, Booth NA and Grebogi C (2009) Chaotic advection in blood flow. Physical Review E 80(1): 016213.

Scheuring I, Károlyi G, Péntek Á, Tél T and Toroczkai Z (2000) A model for resolving the plankton paradox: coexistence in open flows. Freshwater Biology 45(2): 123-132.

Solomon S (1999) Stratospheric ozone depletion: a review of concepts and history. Reviews of Geophysics 37(3): 275-316.

Stroock AD, Dertinger SKW, Ajdari A, Mezic I, Stone HA and Whitesides GM (2002) Chaotic mixer for microchannels. Science 295(5555): 647-651.

Tél T and Gruiz M (2006) Chaotic Dynamics - An Introduction Based on Classical Mechanics. Cambridge University Press, Cambridge.

Tél T, de Moura A, Grebogi C and Károlyi G (2005) Chemical and biological activity in open flows: a dynamical system approach. Physics Reports 413(2-3): 91-196.

Tew Kai E, Rossi V, Sudre J, Weimerskirch H, López C, Hernández-García E, Marsac F and Garcon V (2009) Top marine predators track Lagrangian coherent structures. Proceedings of the National Academy of Sciences of the USA 106(20): 8245-8250.

Toggweiler JR and Russell J (2008) Ocean circulation in a warming climate. Nature 451(7176): 286-288.

Voth GA, Haller G and Gollub JP (2002) Experimental measurements of stretching fields in fluid mixing. Physical Review Letters 88(25): 254501.

Vreugdenhil CB (1994) Numerical Methods for Shallow-water Flow. Kluwer Academic, Dordrecht, the Netherlands.

Whitehead PG, Wilby RL, Battarbee RW, Kernan M and Wade AJ (2009) A review of the potential impacts of climate change on surface water quality. Hydrological Sciences Journal 54(1): 101-123.

Wolff GT (2008) Mesoscale and synoptic scale transport of aerosols. Annals of the New York Academy of Sciences 338(1): 379-388

\section{What do you think?}

To discuss this paper, please email up to 500 words to the editor at journals@ice.org.uk. Your contribution will be forwarded to the author(s) for a reply and, if considered appropriate by the editorial panel, will be published as discussion in a future issue of the journal.

Proceedings journals rely entirely on contributions sent in by civil engineering professionals, academics and students. Papers should be 2000-5000 words long (briefing papers should be 1000-2000 words long), with adequate illustrations and references. You can submit your paper online via www.icevirtuallibrary.com/content/journals, where you will also find detailed author guidelines. 